\title{
Estudos de Validação: Qual Análise Utilizar?
}

\section{Prezado Editor Chefe da RBME,}

O artigo intitulado: Validação da equação de Brzycki para a estimativa de 1-RM no exercício supino em banco inclinado (vol. 13, no 1 - Jan/Fev, 2007) apresenta resultados importantes quanto às diferenças entre estimativas de valores médios e associação linear entre os valores produzidos pela equação de Brzycki e pelo teste padrão para determinação da carga de uma repetição máxima (1-RM). Nesse estudo, utilizaram-se na análise estatística o teste $t$ de Student pareado, o coeficiente de correlação de Pearson, o erro padrão de estimativa (EPE), erro total (ET) e erro constante (EC). Entre os resultados, não foram encontradas diferenças significativas entre os valores médios do teste de 1-RM e os valores médios preditos a partir desta equação, uma correlação de 0,99 ( $p<0,05)$, EPE de 2,42kg $(\approx 3,4 \%)$, ET de 1,55kg $(\approx 2,2 \%)$ e EC de $0,22 \mathrm{~kg}(\approx 0,3 \%)$.

A validade de um instrumento, de forma geral, refere-se à capacidade deste em medir realmente aquilo que se deseja mensurar sem a presença de viés. O viés representa o erro sistemático que ocorre no desenho ou mesmo durante o estudo, podendo comprometer as conclusões deste. Diferentemente do erro sistemático, o erro aleatório representa a diferença entre a estimativa obtida na amostra e o parâmetro real na população de referência (SZKLO e NIETO, 2006). Fica claro, então, que toda análise envolvida em estudos com essa característica deve direcionar suas conclusões a fim de avaliar o impacto desses erros nas estimativas.

A fim de contribuir com o debate sobre a utilização de técnicas empregadas para análise de validação de testes, a presente resenha tem por objetivo apontar importantes limitações na estratégia de medida de validade de critério utilizada no estudo, além de sugerir a utilização de outra ferramenta de análise.

Inicialmente, o teste té uma análise estatística cujo objetivo é testar a hipótese de diferença entre duas médias (i.e., 1-RM vs predição) sob a hipótese nula de que elas são iguais, a partir da probabilidade do erro tipo 1. Em estudos de validação, esse teste pode ser uma ferramenta de determinação de erros sistemáticos importantes (ATKINSON e NEVILL, 1998), porém o artigo não discute essa característica.

Além disto, Bland e Altman (1995) afirmam que a detecção de diferenças significativas através do teste t está constantemente associada à variação aleatória entre os testes, já que não são raras as situações nas quais essa análise não é capaz de detectar diferenças significativas em dois conjuntos de dados com médias similares, porém com elevada variação aleatória intrassujeitos.

A comparação de médias pode ser utilizada como análise inicial, contudo, essa estratégia não permite a realização de qualquer inferência sobre a concordância dos valores individuais (limitação também encontrada no cálculo do EC, que representa a diferença entre a média do teste de 1-RM e a média da predição).

A correlação de Pearson foi utilizada para avaliar o grau de associação entre as medidas. Essa técnica expressa um índice de associação linear entre duas variáveis contínuas e vem sendo apresentada como uma usual medida de validade (vide a discussão do artigo). Entretanto, essa estatística não é capaz de determinar diferenças sistemáticas, além de ser consideravelmente sensível à amplitude e aos valores extremos da variável em análise (BATES et al., 1996). Assim sendo, em um grupo com características variadas (i.e., grande variabilidade intersujeitos), a correlação de Pearson poderia causar uma superestimativa na associação e possíveis conclusões errôneas quanto à validade do instrumento (ATKINSON e NEVILL, 1998).
Com base nos dados deste estudo, pode-se observar para a equação de Brzycki, um valor médio de 70,6kg, um desvio padrão de 18,9kg e um coeficiente de variação de aproximadamente $26,8 \%$, variação esta considerada elevada e em grande medida um importante determinante para a alta correlação encontrada.

O erro padrão da estimativa (EPE) foi outra medida calculada para avaliar a utilização da equação de Brzycki; entretanto, considerando-se as diversas formas para o seu cálculo e mesmo que não apresentada no método do artigo, conclui-se que essa medida se baseou no valor de correlação previamente apresentada:

$$
\mathrm{EPE}=\mathrm{S} \sqrt{1-\rho^{2}}
$$

Onde S é o desvio padrão e $\rho$ é a correlação de Pearson obtida.

Por conseguinte, tal variável pode estar viesada, uma vez que o valor da correlação de Pearson pode estar superestimado, o que minimizaria o erro associado à predição de 1-RM pela equação.

O método proposto por Bland \& Altman (1983) reconhece essas limitações. A a partir da utilização de limites de concordância, desenvolveram uma estratégia para comparar o quanto um método difere de outro e se a diferença entre as medidas está relacionada com a magnitude da mesma (heterocedasticidade dos dados). Além disso, outra vantagem importante apresentada pelos autores é a possibilidade de analisar a diferença entre os métodos em cada um dos sujeitos que compõem a amostra.

Essa abordagem baseia-se na construção de um gráfico de dispersão cujas médias e diferenças entre os métodos encontram-se no eixo das abscissas e das ordenadas, respectivamente. Adicionalmente, utilizam-se eixos como limites ao eixo da abscissa, que determinam a diferença média entre os métodos (i.e., erro sistemático) e o desvio padrão das diferenças (i.e., erro aleatório ao redor dessa média). Essa técnica tem se mostrado altamente informativa e requer pouca sofisticação nas rotinas computacionais.

Em síntese, ao invés de utilizar diversas análises, cada uma com importantes limitações, sugere-se a utilização do gráfico de Bland \& Altman como estratégia de análise em estudos de validação que avaliam variáveis contínuas.

Szklo M, Nieto FJ. Epidemiology Beyond the Basics. Jones and Bartlett Publishers. 2nd ed., 2006. Bland JM, Altman DG. Comparing two methods of clinical measurement: a personal history. Int J Epidemiol. 1995;24 Suppl 1:S7-14.

Bates BT, Zhang S, Dufek JS, Chen FC. The effects of sample size and variability on the correlation coefficient. Med Sci Sports Exerc. 1996 Mar;28(3):386-91

Atkinson G, Nevill AM. Statistical methods for assessing measurement error (reliability) in variables relevant to sports medicine. Sports Med. 1998 Oct;26(4):217-38. Review.

Bland JM, Altman DG. Measurement in Medicine: the Analysis of Method Comparison Studies. The Statistician. 1983, 32:307-17.

\section{Autores}

Leonardo Gonçalves Ribeiro

Mestre em Ciência da Motricidade Humana (UCB/RJ)

Pro Health \& Performance

Letícia de Oliveira Cardoso

Mestre em Saúde Coletiva pelo Instituto de Medicina Social da UERJ,

Doutoranda em Saúde Pública na Escola Nacional de Saúde Pública/Fiocruz,

Departamento de Epidemiologia e Métodos Quantitativos em Saúde/ENSP/Fiocruz 


\section{Resposta}

Fomos convidados para nos pronunciar com relação a uma carta submetida ao Editor Chefe da RBME pelos pesquisadores Leonardo Gonçalves Ribeiro e Letícia de Oliveira Cardoso, intitulada "Estudos de Validação: Qual análise utilizar?", onde o nosso artigo publicado nessa revista (Validação da equação de Brzycki para a estimativa de 1-RM no exercício supino em banco horizontal, v.13, n.1, p.47-50, 2007) foi utilizado como referência.

Os elementos apresentados pelos dois pesquisadores chamam a atenção para a limitação no tratamento das informações apresentadas no artigo em questão, apontando a necessidade da inclusão de critérios de análise adicionais (especificamente, a plotagem de Bland-Altman para análise de concordância) em estudos de validação, como aquele utilizado como exemplo.

Concordamos com a crítica apresentada ao longo da carta de que a apresentação de tais informações poderia fortalecer ou enfraquecer a análise dos dados apresentados em nosso trabalho e, desse modo, nos propusemos a refinar a análise apresentada, incluindo não somente as informações produzidas pela plotagem de Bland-Altman, mas também outros critérios de performance que, mais recentemente, vêm sendo sugeridos na literatura para estudos de validação, com ênfase na análise de concordância, tais como: avaliação do slope e do intercepto gerados pela análise de correlação de Pearson frente à reta de identidade e uma análise de tendência com base nos dados da plotagem de Bland-Altman.

Em nosso estudo, os resultados a partir da análise de regressão entre o teste de 1-RM no supino em banco horizontal e a estimativa determinada pela equação de Brzycki indicaram que o valor do slope e do intercepto não diferiram da linha de identidade (slope $=1$ e intercepto $=0 ; P>0,05$ ), o que fortalece os valores da correlação por nós relatados $(r=0,99 ; P<0,05)$. Essas informações suplantam em parte as limitações da utilização da análise de correlação, de forma isolada, para testar a validade de um determinado método frente a um método adotado como referência, visto que a força da correlação não indica concordância entre dois métodos.

Com relação à plotagem de Bland-Altman, nossos dados indicaram que a estimativa dos valores de 1-RM pela equação de Brzycki apresentaram um baixo viés (bias $=-0,3)$ com limites de concordância bastante aceitáveis $(4,9$ a $-5,5 \mathrm{~kg})$ indicando que os resultados poderiam estar subestimados em no máximo 6,9\% ou superestimados em no máximo 7,8\% em 96\% da amostra investigada $(n=48)$.

Ao analisarmos a existência de uma possível tendência associada aos maiores ou menores níveis de força dos sujeitos que compuseram a amostra, os resultados indicaram um $r=-0,18$ ( $P$ $=0,22$ ), refutando a hipótese de que a grande variabilidade intersujeitos poderia estar comprometendo os achados, com base nas informações originais apresentadas no manuscrito.

Portanto, agradecemos a oportunidade que nos fora oferecida pela RBME para reforçarmos ainda mais os achados apresentados pelo nosso estudo de que a equação de Brzycki parece, realmente, ser uma alternativa bastante atraente para a estimativa dos valores de 1-RM no exercício supino em banco horizontal, a partir da execução de testes submáximos de 7 a 10-RM, em homens adultos sedentários ou moderadamente ativos.

Prof. Dr. Edilson Serpeloni Cyrino

Departamento de Educação Física (DEF)

Centro de Educação Física e Esporte (CEFE)

Universidade Estadual de Londrina (UEL)

Paraná - Brasil

Tel./Fax: (43) 33714772.

E-mail: emcyrino@uel.br 